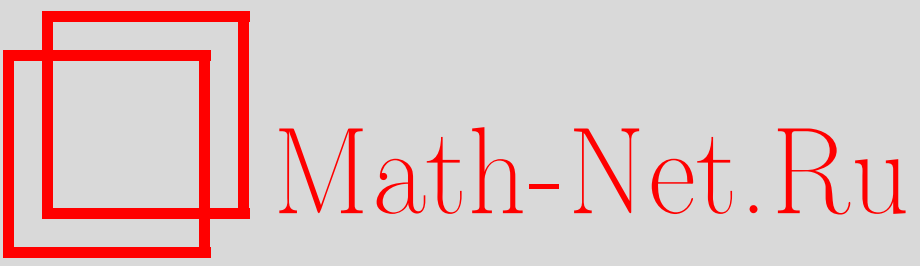

В. А. Зорич, В. М. Кесельман, Изопериметрическое неравенство на субримановых многообразиях конформно-гиперболического типа, УМH, 2000, том 55, выпуск 6, 137-138

DOI: https://doi.org/10.4213/rm343

Использование Общероссийского математического портала Math-Net.Ru подразумевает, что вы прочитали и согласны с пользовательским соглашением

http://www. mathnet.ru/rus/agreement

Параметры загрузки:

IP: 3.89 .197 .203

26 апреля 2023 г., 13:38:16 


\title{
ИЗОПЕРИМЕТРИЧЕСКОЕ НЕРАВЕНСТВО НА СУБРИМАНОВЫХ МНОГООБРАЗИЯХ КОНФОРМНО-ГИПЕРБОЛИЧЕСКОГО ТИПА
}

\author{
В. А. ЗОРИЧ, В. М. КЕСЕЛЬМАН
}

1. Введение. В этой заметке мы имеем в виду отметить, что основные резултаты нашей работы [1] о каноническом виде изопериметрического неравенства на римановых многообразиях конформно-гиперболического типа распространяются и на субримановы многообразия. Точные формулировки будут даны ниже в разделе 5 после введения необходимых понятий.

Как известно, изопериметрическое неравенство - это соотношение вида

$$
P(V(D)) \leqslant S(\partial D)
$$

между объемом $V(D)$ области $D$ и площадью $S(\partial D)$ ее границы, где $P$ - функция, называемая изопериметрической функцией пространства.

Нас интересует канонический вид изопериметрического соотношения в конформном классе метрики многообразия, точнее, вопрос о том, к какому каноническому виду приводится изпериметрическое неравенство конформными заменами метрики многообразия.

2. Субриманово многообразие. Гладкое многообразие $M$ вместе с поляризацией $H \subset T M$ (т.е. подрасслоением $H$ касательного расслоения $T M)$ и римановой структурой $g$ на $H$ называют субримановым многообразием и обозначают через $(M, H, g)$ или $(M, H)$ (см. [2]).

Поляризация выделяет множество направлений (касательных векторов), обычно называемых горизонтальными. Кусочно-гладкие кривые на $M$ называют горизонтальныцми, если касательные к ним векторы горизонтальны, т.е. лежат в $H$.

Метрика Карно-Каратеодори ( $C$ - $C$-метрика), ассоциированная с предполагаемой неголономной (неинтегрируемой) поляризацией $H$ и римановой структурой $g$ на $H$, определяется через нижнюю грань $g$-длин горизонтальных кривых, соединяющих точки.

Метрическую (хаусдорфову) размерность многообразия $M$ как $C$ - $C$-пространства будем в дальнейшем обозначать через $m$. Она больше топологической размерности $n$ многообразия $M$, если $H \neq T M$. Например, для группы Гейзенберга, рассматриваемой как $C$ - $C$-пространство, $m=n+1$. Для собственно риманова многообразия, конечно, $m=n$.

3. Конформная емкость. Горизонтальный градиент $\nabla f=\nabla_{H, g} f$ функции $f$ на $(M, H, g)$ определяется как единственный горизонтальньй вектор такой, что

$$
\langle\nabla f, X\rangle_{g}=X f
$$

для всех горизонтальных векторов $X$.

Здесь $\langle\cdot, \cdot\rangle_{g}$ - скалярное произведение относительно римановой структуры $g$ на $H$, а $X f$ производная Ли функции $f$ по вектору $X$.

Пусть $G$ - область на открытом субримановом многообразии $(M, H)$, а $C$ - отличный от точки континуум (связный компакт) в $G$. Пару $(C, G)$ часто называют конденсатором.

Конформной емкостью конденсатора $(C, G)$ называется величина

$$
\operatorname{cap}(C, G)=\inf \int_{G}|\nabla f|^{m} d v,
$$

где нижняя грань берется по всем гладким финитным в $G$ функциям $f$ таким, что $f \equiv 1$ на $C$ и $0 \leqslant f \leqslant 1$ в $G$. При этом интегрирование ведется по $m$-мере Хаусдорфа.

Произвольную риманову метрику $\widetilde{g}$ на $H$ называют конформно-әквивалентной или конформной метрике $g$, если $\widetilde{g}=\lambda^{2} g$ на $H$, где $\lambda$ - некоторая положительная регулярная функция на $M$. Легко видеть, что величина сар $(C, G)$ инвариантна относительно конформных замен исходной метрики субриманова многообразия.

Работа выполнена при поддержке Российского фонда фундаментальных исследований (грант № 99-01-01179). 
4. Конформный тип субриманова многообразия. Некомпактное субриманово многообразие $(M, H, g)$ будем назьвать многообразием конформно-параболического типа, если конформная емкость его абсолюта равна нулю, т.е. $\operatorname{cap}(C, M)=0$ для любого невырожденного континуума $C \subset M$; если же эта емкость положительна, то многообразие $(M, H, g)$ будем называть многообразием конформно-гиперболического типа.

Такое разделение субримановых многообразий по указанным конформным типам, очевидно, инвариантно относительно конформных замен метрики многообразия.

\section{5. Формулировка основных результатов.}

Теорема. Субриманово некомпактное и без края многообразие $(M, H, g)$ имеет конформно-гиперболический тип тогда и только тогда, когда существует конформно-эквивалентная метрике $g$ риманова метрика $\widetilde{g}$, в которой выполняется изопериметрическое неравенство:

$$
\widetilde{V}(D) \leqslant \widetilde{S}(\partial D)
$$

для всех регулярных областей $D \subset M$, обвем $\widetilde{V}(D)$ которых больие фиксированного (произвольно малого) положительного числа.

Здесь под регулярной областью понимается область с кусочно-гладкой границей, имеюшая конечный $m$-мерный $\widetilde{g}$-объем $\widetilde{V}(D)$ и конечную $(m-1)$-мерную $\widetilde{g}$-меру Хаусдорфа $\widetilde{S}(\partial D)$ границы области.

Таким образом, в конформном классе метрики субриманова многообразия конформно-гиперболического типа изопериметрическая функция приводится к тому же линейному виду $P(x)=x$, что и в пространстве Лобачевского.

ДополнениЕ 1. Указанное в теореме неравенство (*) является асимптотически точным в том смысле, что отношение величин $\widetilde{V}(D)$ и $\widetilde{S}(\partial D)$ может быть сколь угодно близким к единице для регулярных областей сколь угодно больиого $\widetilde{g}$-обгема.

ДополнениЕ 2. Указанную в теореме метрику $\widetilde{g}$ можно выбрать так, что оббем геодезического шара радиуса $r$ в этой метрике имеет порядок величинь $e^{r}$ при $r \rightarrow+\infty$.

Напомним [3], что изопериметрической размерностью многообразия бесконечного объема называется точная верхняя грань всех чисел $d>0$, для каждого из которых функция $P(x)=$ $x^{\frac{d-1}{d}}$ (при всех $x>\varepsilon>0$ ) является изопериметрической функцией многообразия. Точную верхнюю грань изопериметрических размерностей многообразия по всем метрикам, конформным исходной метрике многообразия, будем называть конформной изопериметрической размерностью многообразия (см. [4]). Используя сформулированную выше теорему, можно получить

СледСтвиЕ. Субриманово многообразие $(M, H, g)$ имеет конформно-гиперболический тип тогда и только тогда, когда его конформная изопериметрическая размерность равна $+\infty$.

\section{СПИСОК ЛИТЕРАТУРЫ}

[1] Зорич В. А., Кесельман В. М. // УМН. 1999. Т. 54. №3. С. 165-166. [2] Bellaiche A. (ed.) // Sub-Riemannian Geometry: Proceedings of the satellite meeting of the 1st European congress of mathematics (Paris, June 30-July 1, 1992). Basel: Birkhäuser, 1996. (Progr. Math. V. 144.) [3] Gromov M. with appendices by Katz M., Pansu P., Semmes S. Metric Structures for Riemannian and Non-Riemannian Spaces. Boston: Birkhäuser, 1999. [4] Зорич В. А., Кесельман В. М. // УМН. 1999. Т. 54. №4. С. 171-172.

Московский государственный

Принято редколлегией университет им. М. В. Ломоносова

03.10 .2000 